Published in final edited form as:

Diabetes Res Clin Pract. 2013 May ; 100(2): 265-271. doi:10.1016/j.diabres.2013.03.013.

\title{
Fructose Intake and Cardiovascular Risk Factors in Youth with Type 1 Diabetes: SEARCH for Diabetes in Youth Study
}

\author{
Sarah C. Couch, Ph.D., RD ${ }^{1}$, Jamie L. Crandell, Ph.D², Amy S. Shah, MD ${ }^{3}$, Lawrence M. \\ Dolan, MD $^{3}$, Anwar T. Merchant, Ph.D ${ }^{4}$, Angela D. Liese, Ph.D. ${ }^{4}$, Jean M. Lawrence, ScD, \\ MPH, MSSA ${ }^{5}$, Catherine Pihoker, MD $^{6}$, and Elizabeth J. Mayer-Davis, PhD, MSPH, RD ${ }^{7}$ \\ ${ }^{1}$ Department of Nutritional Sciences, University of Cincinnati Medical Center, Cincinnati, $\mathrm{OH}$ \\ 45267-0394
}

${ }^{2}$ School of Nursing and Department of Biostatistics, University of North Carolina, Chapel Hill, NC, 27599

${ }^{3}$ Department of Endocrinology, Cincinnati Children's Hospital Medical Center, Cincinnati, OH 45267

${ }^{4}$ Department of Epidemiology and Biostatistics and Center for Research in Nutrition and Health Disparities, Arnold School of Public Health, University of South Carolina, Columbia, SC, 29208

${ }^{5}$ Department of Research \& Evaluation, Kaiser Permanente Southern California, Pasadena, CA, 91101

${ }^{6}$ Department of Endocrinology, University of Washington Pediatrics, Children's Hospital Endocrinology Clinic, Seattle, Washington 98145

${ }^{7}$ Department of Nutrition and Department of Medicine, University of North Carolina, Chapel Hill, NC, 27599

\section{Abstract}

\begin{abstract}
Aims-High consumption of dietary fructose has been shown to contribute to dyslipidemia and elevated blood pressure in adults, but there are few data in youth, particularly those at greater risk of cardiovascular disease (CVD). The aim of this study was to examine the association between fructose intake and CVD risk factors in a diverse population of youth with type 1diabetes (T1D).
\end{abstract}

Methods-This was a cross-sectional analysis of data from the SEARCH for Diabetes in Youth study, including 2085 youth ages 10-22 years with T1D, of which $22 \%$ were racial/ethnic

\footnotetext{
(C) 2013 Elsevier Ireland Ltd. All rights reserved.

Corresponding author address: Sarah C. Couch, Ph.D., RD. 3202 Eden Avenue, French Building East, Room 363B, University of Cincinnati Medical Center, Cincinnati, OH 45267-0394; Telephone: 513-558-7504; Fax: 513-558-7500; Sarah.Couch@uc.edu.

Publisher's Disclaimer: This is a PDF file of an unedited manuscript that has been accepted for publication. As a service to our customers we are providing this early version of the manuscript. The manuscript will undergo copyediting, typesetting, and review of the resulting proof before it is published in its final citable form. Please note that during the production process errors may be discovered which could affect the content, and all legal disclaimers that apply to the journal pertain.

Financial disclosures: none to report

Conflict of Interest Statement: The authors declare that they have no conflict of interest.
} 
minority and $50 \%$ were female. A semi-quantitative food frequency questionnaire was used to assess intake.

Results-Median daily fructose consumption was $7.9 \%$ of total calories. Fructose intake was positively associated with triglycerides ( $\mathrm{p}<.01$ ), but not with total cholesterol, LDL-cholesterol, HDL-cholesterol, or blood pressure after adjustment for physical activity and socio-demographic, clinical, and dietary covariates. An increase in fructose intake of 22 grams (equivalent to a $12 \mathrm{oz}$. can of soda) was associated with a $23 \%$ higher odds of borderline/ high versus low triglycerides $(\mathrm{p}<.005)$.

Conclusion-These data suggest that children with T1D should moderate their intake of fructose, particularly those with borderline or high triglycerides.

\section{Keywords}

Fructose; cardiovascular risk factors; triglycerides; adolescents; type 1 diabetes

\section{Introduction}

Youth with diabetes are at an increased risk for cardiovascular disease (CVD) (1). Diabetesassociated CVD risk factors include elevated triglycerides, low HDL-cholesterol, high LDLcholesterol, and elevated blood pressure, which persist despite improved glycemic control (2). Current dietary guidelines aimed to reduce CVD risk factors in persons with diabetes recommend lowering dietary fat and replacing saturated fat with carbohydrate. However the type of carbohydrate used as a substitute for fat in persons with diabetes may mitigate any benefit (3).

Fructose is a monosaccharide found naturally in fruits. It is also a component of added sugars found in sweetened beverages and processed snacks. In youth, fructose intake has risen sharply with the increased use of high fructose corn syrup (HFCS) as a sweetening agent (4). Nearly half of the fructose consumed by adolescents comes from sweetened beverages (5). While high fructose benefits glucose control (6), high fructose intakes could have potentially negative consequences on CVD risk factors, as high intakes of the carbohydrate are known to increase fatty acid and triglyceride synthesis (7). Diabetes may exacerbate this effect as inadequate insulinization or insulin resistance may increase triglyceride synthesis and reduce triglyceride clearance (8). Additionally, both diabetes and high fructose consumption may elevate serum uric acid (9), which is a risk factor for hypertension (10).

Studies linking dietary fructose with CVD risk factors in diabetes have focused on the adult population. Disparate findings have been reported in this regard, with some studies showing positive associations between higher fructose consumption and plasma lipids $(11,12)$ and blood pressure (13) and others showing no relationship $(14,15)$. In children with diabetes, one small, short-term feeding trial was done showing no significant effect of high dietary fructose feeding on plasma lipids (16). Confirmation of this finding is warranted.

Little is known about recent consumption patterns of fructose in children with diabetes. Further, no well-powered studies have examined whether fructose intake in children with 
diabetes alters CVD risk factors. Toward this purpose, data from the SEARCH for Diabetes in Youth study were used to examine fructose intake in youth with type 1diabetes (T1D) and determine whether intake of the monosaccharide is associated with serum lipids, lipoproteins, and blood pressure.

\section{Subjects}

The SEARCH for Diabetes in Youth study is an ongoing multi-center, observational study with the goal of describing the epidemiology of childhood diabetes according to race/ ethnicity, age, sex, and diabetes type. The study began ascertainment of all existing (prevalent) cases of physician diagnosed diabetes mellitus (DM) in youth <20 years of age in calendar year 2001 and all newly diagnosed (incident) cases during subsequent years. SEARCH has six centers located in Ohio, Washington, South Carolina, Colorado, Hawaii and California. A detailed description of the SEARCH study design and methods has been published elsewhere (17).

The present study was a cross-sectional analysis of data from the prevalent and 2002-2005 incident study cohort participants. Data were available on 2,586 children 10-20 years of age with T1D who participated in the SEARCH baseline exam and dietary assessment at least 3 months after their diabetes diagnosis. Participants were then excluded from analysis for the following reasons: reporting an atypical week where they ate "a lot more" or "a lot less than usual on the baseline food frequency questionnaire (FFQ) $(n=112)$; reported dietary intake in excess of calorie cut-point (>7000 kcal/day, $\mathrm{n}=2$ ); not having baseline blood drawn in the fasted state $(\mathrm{n}=211)$; having blood lipid variables otherwise unavailable $(\mathrm{n}=161)$; or hypertriglyceridemia (TG>400 mg/dl, $\mathrm{n}=15$ ). After all exclusions were made, the final sample was comprised of 2,085 youth with T1D.

\section{Materials and Methods}

The study was reviewed and approved by the local Institutional Review Boards at each center. Parents of participants under age 18 years at the time of data collection provided written informed consent and the participant provided assent; all participants aged 18 years or older provided written informed consent. All participants in this cross-sectional sample completed an initial patient survey (online or by telephone interview) to collect information on race/ethnicity, diabetes type, and preliminary treatment information. Participants also completed an in-person visit (baseline exam) consisting of a physical examination (anthropometry, blood pressure), laboratory work (fasting blood work for HbAlc and lipids), and the administration of several questionnaires to collect information on medical history, physical activity, and dietary intake. All measures were conducted by trained, certified staff in accordance with standardized study protocols (available at www.searchfordiabetes.org). A more detailed description of the measures considered in the present study is described below.

\section{Assessment of Dietary Intake}

A modified Block Kid's FFQ was used to assess dietary intake in this multi-cultural study. A description of the modified questionnaire, hereafter referred to as the SEARCH FFQ, and 
validation are described elsewhere $(18,19)$. In brief, the SEARCH FFQ is semi-quantitative with approximately 85 food lines and weekly frequency of use categories for each line item. Portion size for each food or beverage are assessed in a manner relevant to that particular item, e.g. number of slices for bread; small, medium and large for food in bowls, number of pieces of fruit. Questionnaire items also addressed atypical intake, use of dietary supplement, use of modified food products (e.g., low fat), and frequency of eating out. The FFQ was self-administered by study participants after careful instruction by staff. If it was apparent that the subject would have difficulty completing the form, a staff member conducted the form administration as an interview. The nutrient and portion-size database for this instrument was established using the Nutrition Data Systems for Research database (version 4.05/33, 2002, Nutrition Coordinating Center, University of Minnesota, Minneapolis, MN) and industry sources. Energy, macronutrients, micronutrients and food group servings were quantified. For these analyses sugars were reported as free fructose, sucrose, total fructose (free fructose $+1 / 2$ the intake of sucrose), free fructose food subgroups (vegetables, fruit $\&$ fruit juice), and added fructose food subgroups (sweets \& desserts, and sugar-sweetened beverages).

\section{Outcomes}

Blood lipids - Fasting (minimum 8 hours) blood samples were obtained under conditions of metabolic stability, defined as no episode of diabetic ketoacidosis during the previous month. Specimens were processed at the site and shipped within 24 hours to the Northwest Lipid Metabolism and Diabetes Research Laboratories in Seattle, Washington. Measurement of plasma cholesterol, triglyceride, and HDL cholesterol were performed on a Hitachi 917 autoanalyzer (Boehringer Mannheim Diagnostics, Indianapolis, IN). LDL cholesterol was calculated by the Friedewald equation (20). Blood pressure - Blood pressure measurements were performed using a standard mercury sphygmomanometer with 1 of 5 cuff sizes chosen on the basis of the circumference of the participant's arm. The average of 3 blood pressure measurements was used in the analyses.

\section{Covariates}

Demographics - adolescents and a parent/guardian self-reported birth date, gender, race/ ethnicity and duration of diabetes. Body mass index - Body weight and height were measured in light indoor clothing without shoes. Measurements were recorded twice and averaged. BMI was calculated as weight in kilograms divided by the square of height in meters. BMI z-scores were determined using the standard CDC approach (21). HbA1c- A1c measurement was made on whole blood samples (obtained after an 8 hour fast) with an automated nonporous ion-exchange highperformance liquid chromatography system (model G-7; Tosoh Bioscience, Montgomeryville, Pennsylvania). HbA1c assays were performed at the Northwest Lipid Metabolism and Diabetes Research Laboratories, University of Washington. Physical Activity - Participants were asked the average number of days in a typical week that they participated in physical activity for at least 20 minutes that made them sweat or breathe hard and were then categorized as physically inactive ( $0-2$ days/week) or physically active (3-7 days/week). The question was derived from the national Youth Risk Behavior Survey (22). 


\section{Statistical Analyses}

The initial analyses included a description of the bivariate relationships between fructose and other study variables. In order to make these relationships more clinically relevant, the percent of energy derived from fructose (fructose density) was used here. Characteristics of the study population were compared across quartiles of fructose density. Analysis of variance was used to assess bivariate associations between fructose density and continuous variables, and chi-square tests were used for categorical variables. Triglycerides were logtransformed to improve normality. In the regression models, adjustment for energy was done differently. As recommended by Willett, Howe and Kushi (23), the primary independent/ predictor variable of interest was total fructose intake in grams per day, with additional model adjustment for total energy intake. Multiple regression models were used to assess multivariate relationships between CVD risk factors and fructose intake (g/day) with adjustment for total calories and other covariates. Analyses examining the appropriateness of using fructose as a categorical variable were performed, and model assumptions were better met with fructose as a continuous predictor. Initial models adjusted for energy intake only. Final models adjusted additionally for age, race, gender HbA1c, BMI z-score, T1D duration, physical activity, intake of other carbohydrates (total carbohydrate - total fructose), and total dietary fiber. Final models also adjusted for other dietary nutrients that were found to modify the beta coefficient $>10 \%$ thus indicating confounding. These differed by outcome and are as follows: 1) intake of saturated fat, monounsaturated fat, and trans fat in the models for plasma total, LDL and HDL-cholesterol; 2) intake of trans fat in the model for log-triglycerides; and 3) intake of calcium, magnesium, potassium and sodium in the models for blood pressure. Residual plots for the adjusted model were created to examine the appropriateness of assuming a linear relationship between log-triglycerides and fructose intake, and no violations of the linearity assumption were evident. As a further sensitivity analysis, the models were examined for evidence of a possible threshold effect of fructose by including fructose as a categorical variable (quartiles) rather than continuous. No evidence of a threshold effect was found.

For regression models that provided evidence of an association between fructose intake and CVD risk outcomes, proportional odds models were fit to characterize the relationship between fructose intake and membership in established risk categories for these outcomes. The appropriateness of the proportional odds assumption was tested and found to be acceptable. Statistical analyses were performed with SAS software (version 9.1, SAS Institute, Cary, North Carolina). P values $<0.05$ were considered to be statistically significant.

\section{Results}

Median fructose intake was $34.6 \mathrm{~g} /$ day (Interquartile range $(\mathrm{IQR})=22.7$ to $51.0 \mathrm{~g} / \mathrm{day}$, mean $=40.5 \mathrm{~g} / \mathrm{day}$ ). This intake accounted for a median fructose density of $7.9 \%$ of calories $(\mathrm{IQR}=5.8$ to $10.3 \%$, mean $=8.4 \%)$. Figure 1 shows the distribution of fructose density among participants. Median total daily energy intake was $1757 \mathrm{kcal}$ (IQR=1354 to 2330 $\mathrm{kcal}$, mean $=1925 \mathrm{kcal})$. 
Table 1 provides clinical characteristics and cardiovascular risk factors for the cohort and by quartile of fructose density ( $\% \mathrm{kcal})$. Participants who were female or African American consumed a higher fructose density than individuals in their respective comparison groups $(p<.05, p<.01$ respectively). Fructose intake was significantly related to age $(p<.01)$, with the first and fourth quartiles being slightly older than the second and third quartiles. Participants who had a longer duration of diabetes consumed a higher fructose density than those with shorter disease duration $(\mathrm{p}<.01)$. Those in the second and third quartiles of fructose intake engaged in more physical activity $(\mathrm{p}<.05)$. Higher fructose density was associated with higher intakes of dietary fiber, free fructose, sucrose, fruit/ fruit juice, sweets and desserts, sugar-sweetened beverages, and other carbohydrates ( $\mathrm{p}<.01$ for all). No significant associations were seen between fructose density and BMI-z score, HbA1c or vegetable intake.

Fructose density was significantly associated with plasma triglycerides $(\mathrm{p}<.01)$. Participants in the top quartile of fructose density had higher triglycerides than those in lower three quartiles. Participants in the first and fourth quartiles of fructose density also had higher SBP and DBP ( $p<.01, \mathrm{p}<.05$ respectively). No significant associations were seen between fructose density and total, LDL or HDL-cholesterol.

To examine the association between fructose intake (g/day) and CVD risk factors, multiple regression models were fit adjusting only for total energy intake (kcal) (Model 1), and then for total energy intake and additional covariates (Model 2). Fructose intake was significantly positively associated with log-triglycerides in both models ( $\mathrm{p}<.01$ in both) (Table 2). This finding was upheld whether total fructose or free fructose was used as the independent variable in the model (data not shown for free fructose). Triglycerides were modeled on the log scale, so an increase in fructose intake of 22 grams, which is the amount of fructose in a 12 ounce can of soft drink (24) was associated with a $4 \%$ increase in triglycerides. Fructose intake was not associated with total, LDL or HDL-cholesterol or BP in either model.

In order to further quantify the relationship between fructose intake and triglycerides, a proportional odds model was used to predict membership in a triglyceride risk category by fructose intake. Risk categories based on established triglyceride cut-offs (8) were low $(<1.30 \mathrm{mmol} / \mathrm{L}, \mathrm{N}=1766)$, borderline $(1.3-1.68 \mathrm{mmol} / \mathrm{L}, \mathrm{N}=164)$, and high $(>1.68 \mathrm{mmol} / \mathrm{L}$, $\mathrm{N}=155)$. After controlling for covariates, fructose was found to be significantly associated with an increased triglyceride risk (Beta $=0.0095, \mathrm{SE}=.0034, \mathrm{p}=.005$ ). This effect size was interpreted by converting the beta coefficient into an odds ratio and determining the odds of membership in a higher versus a lower triglyceride risk category. Based on these calculations an increase in fructose intake of $22 \mathrm{~g}$ was associated with an odds ratio of 1.23 (CI: 1.06, 1.43). In other words, an increase in fructose intake of a 12 ounce soft drink was associated with a $23 \%$ higher odds of membership in a higher triglyceride risk group.

\section{Discussion}

The major new finding of the present study is that higher fructose consumption was associated with greater plasma triglyceride levels in adolescents with T1D independent of many other potential confounders. An increase in fructose intake equivalent to one 12 ounce 
can of soda daily was associated with $23 \%$ higher odds of borderline/ high versus low triglycerides. The fructose triglyceride association in T1D youth fits with proposed mechanisms. Fructose, unlike glucose, does not stimulate insulin secretion from the pancreatic beta cells (25). Fructose is rapidly taken up by the liver, and is metabolized to dihydroxyacetone phosphate and glyceraldehyde (26). The former is used to synthesize the glycerol backbone of triglycerides, while the latter produces acetyl-CoA required for de novo lipogenesis and fatty acid synthesis. These fructose end products are also intermediates in the glycolytic pathway, and are formed in an uncontrolled fashion as fructose enters the pathway below a major point of regulation. Thus, a high postprandial influx of fructose to the liver may increase substrate for triacylglyercol synthesis and secretion, and contribute to elevated blood triglyceride concentrations (26).

Numerous studies have shown that dietary fructose can induce hyperlipidemia in rodents (27-30). For example, Herman et al (28) reported that rats fed a high fructose diet had sustained elevations in serum triglycerides. Triglycerides increased and remained elevated during fructose feeding (100 days) and fell soon after a standard chow diet was introduced. Studies in humans have been less consistent, with positive effects of high fructose intakes on plasma lipids in adults reported in some studies $(10,11)$; but not in others $(13,14)$. In our study, the positive association between fructose and triglycerides is contrary to findings reported from a feeding trial in which fructose was administered to children with T1D at a dose of $20 \%$ of calories. No adverse effects on plasma lipids were observed in this study (16). Inadequate statistical power ( $\mathrm{n}=16)$ and short follow-up period ( 1 week) may have limited the ability to detect group differences in this trial. In a larger, more recent crosssectional study of adolescents without T1D, total fructose consumption was found to be associated with increased plasma triglycerides and LDL-cholesterol and low HDLcholesterol (31). Among healthy Swiss children, dietary fructose was found to be a significant positive predictor of small LDL-particle size (a marker for increase CVD risk) but not plasma triglycerides (32).

Recent related findings from SEARCH found higher plasma triglycerides as well as higher total and LDL cholesterol in adolescents with T1D who drank one glass or more of sugarsweetened beverages compared to those who had none (33). Sugar sweetened beverages are a major food source of fructose in adolescents, and in our cohort, higher fructose intakes were associated with higher intakes of sweetened drinks and desserts, as well as juice and fruit. We did find that models examining triglyceride associations gave the same conclusions whether free or total fructose (free + bound to sucrose) were used; others have suggested different effects of these dietary forms of the monosaccharide on triglyceride metabolism (34). Notably, fructose intakes in our sample were negatively associated with saturated fat intake (data not shown), while soda consumption was positively related to this nutrient (33). This finding suggests that dietary fructose and sugar sweetened beverages, although related, are components of different dietary patterns, and this may account, in part, for the different effects observed on plasma lipids in our cohort.

In our sample of youth 10 years of age and older with T1D, mean intake of total fructose was 40.5 grams/day or $8.4 \%$ of total calories. This is less than the 72.8 grams/day or $12 \%$ of total calories reported nationally among 12 to 18 year olds (5). Several factors may account 
for the lower reported fructose intake in this study including but not limited to differences in dietary data collection methodologies used between studies and response to diabetes related nutrition recommendations that discouraged use of added fructose as a sweetening agent (3). Also, although fructose may benefit glucose control (6), many foods containing fructose, e.g., sugar sweetened beverages and processed grain foods, are high in total carbohydrate and may raise postprandial glycemia. Therefore, youth with T1D may avoid major food sources of fructose in an effort to better manage their diabetes.

While studies in adults have shown associations between fructose and plasma triglycerides generally at intakes $\geq 15 \%$ of total calories (35), in this study among adolescents with T1D, less than $10 \%$ of participants consumed that much fructose. Therefore, adverse effects of fructose on triglycerides may occur across a wider range of intakes than previously observed. Elevated triglycerides track from childhood into adulthood (36) and have been consistently associated with increased CVD, even after adjustment for established coronary risk factors (8). These findings suggest that children and adolescents with T1D should moderate intake of fructose, particularly those with borderline or high triglyceride levels.

Our study has several important strengths including the large sample size of youth with T1D, the racial/ ethnic and geographic diversity of the population, and the variety of outcome and exposure measures obtained from the study sample. Limitations include the cross-sectional nature of the measurements, which allows associations to be identified, but not causality or directionality. FFQ usually tend to over-report dietary intake (37) and the fact that diet intake for only the previous week was collected may not accurately reflect usual long-term consumption of major food sources of fructose.

In summary, fructose intake in adolescents with T1D was positively associated with plasma triglyceride levels. No evidence of a threshold effect of fructose on triglycerides was observed in this study. While this study did not identify the specific food sources of fructose that were problematic in this regard, these findings suggest that persons with diabetes, especially those in high risk triglyceride groups, should moderate fructose consumption to avoid untoward effects on plasma triglycerides.

\section{Acknowledgments}

The SEARCH for Diabetes in Youth Study is indebted to the many youth and their families, and their health care providers, whose participation made this study possible.

Grant Support: SEARCH for Diabetes in Youth is funded by the Centers for Disease Control and Prevention (PA numbers 00097, DP-05-069, and DP-10-001) and supported by the National Institute of Diabetes and Digestive and Kidney Diseases.

Site Contract Numbers: Kaiser Permanente Southern California (U48/CCU919219, U01 DP000246, and U18DP002714), University of Colorado Denver (U48/CCU819241-3, U01 DP000247, and U18DP000247-06A1), Kuakini Medical Center (U58CCU919256 and U01 DP000245), Children's Hospital Medical Center (Cincinnati) (U48/CCU519239, U01 DP000248, and 1U18DP002709), University of North Carolina at Chapel Hill (U48/ CCU419249, U01 DP000254, and U18DP002708-01), University of Washington School of Medicine (U58/ CCU019235-4, U01 DP000244, and U18DP002710-01), Wake Forest University School of Medicine (U48/ CCU919219, U01 DP000250, and 200-2010-35171).

The authors wish to acknowledge the involvement of General Clinical Research Centers (GCRC) at the South Carolina Clinical \& Translational Research (SCTR) Institute, at the Medical University of South Carolina (NIH/ NCRR Grant number UL1RR029882); Children's Hospital and Regional Medical Center (Grant Number 
M01RR00037); Colorado Pediatric General Clinical Research Center (Grant Number M01 RR00069) and the Barbara Davis Center at the University of Colorado at Denver (DERC NIH P30 DK57516); and the Institutional Clinical and Translational Science Award (CTSA), NIH/NCRR at the University of Cincinnati (Grant Number 1UL1RR026314-01).

The contents of this paper are solely the responsibility of the authors and do not necessarily represent the official views of the Centers for Disease Control and Prevention and the National Institute of Diabetes and Digestive and Kidney Diseases.

\section{References}

1. Wadwa RP. Cardiovascular disease risk in youth with diabetes mellitus. Rev Endocr Metab Disord. 2006; 7:197-204. [PubMed: 17160723]

2. Rodriquez BL, Fujimoto WY, Mayer-Davis EJ, Imperator G, Kershnar A, Daniels SR, et al. the SEARCH for Diabetes in Youth Study Group. Prevalence of cardiovascular risk factors in US Children and Adolescents with Diabetes: The SEARCH for Diabetes in Youth Study. Diabetes Care. 2006; 29:1891-1896. [PubMed: 16873798]

3. American Diabetes Association. Nutrition recommendations and interventions for diabetes. Diabetes Care. 2008; 31:S61-S78. [PubMed: 18165339]

4. Marriott BP, Cole N, Lee E. National estimates of dietary fructose intake increased from 1977 to 2004 in the United States. J Nutr. 2009; 139:1228S-1235S. [PubMed: 19403716]

5. Vos MB, Kimmons JE, Gillespie C, Welsh J, Blanck HM. Dietary fructose consumption among US children and adults: The Third National Health and Nutrition Examination Survey. Medscape J Med. 2008; 10:160-169. [PubMed: 18769702]

6. Cozma AI. Effect of Fructose on Glycemic Control in Diabetes. Diabetes Care. 2012; 35:1611-20. [PubMed: 22723585]

7. Sanchez-Lozada LG, Le M, Segal M, Johnson RJ. How safe is fructose for persons with or without diabetes? Am J Clin Nutr. 2008; 88:1189-90. [PubMed: 18996851]

8. Miller M, Stone NJ, Ballantyne C, Bittner V, Criqui MH, Ginsberg HN, Goldberg AC, Howard WJ, Jacobson MS, Kris-Etherton PM, Lennie TA, Levi M, Mazzoni T, Pennathur S. Triglycerides and cardiovascular disease: a scientific statement from the American Heart Association. Circulation. 2011; 123:2292-2333. [PubMed: 21502576]

9. Lanaspa MA, Tapia E, Soto V, Sautin Y, Sanchez-Lozada LG. Uric acid and fructose: potential biological mechanisms. Sem Nephrology. 2011; 31:426-32.

10. Angelopoulos TJ, Lowndes J, Zukley L, Melanson KJ, Nguyen V, Huffman A. The effect of highfructose corn syrup consumption on triglycerides and uric acid. J Nutr. 2009; 139:1242A-5S.

11. Stanhope KC, Brenner AA, Medici V, Nakajima K, Ito Y, Nakano T, Chen G, Fong TH, Lee V, Menorca RI, Keim NC, Havel PJ. Consumption of fructose and high fructose corn syrup increase postprandial triglycerides, LDL-cholesterol and apolipoprotein B in young men and women. J Clin Endocrinology Metab. 2011; 98:E1596-605. [PubMed: 21849529]

12. Bantle JP, Raatz SK, Thomas W, Georgopoulos A. Effects of dietary fructose on plasma lipids in healthy subjects. Am J Clin Nutr. 2000; 72:1128-34. [PubMed: 11063439]

13. Jalas DI, Smits G, Johnson RJ, Chonchol M. Increased fructose associates with elevated blood pressure. J Am Soc Nephrology. 2010; 21:1543-9.

14. Schaefer EJ, Gleason JA, Dansinger ML. Dietary fructose and glucose differentially affect lipid and glucose homeostasis. J Nutr. 2009; 139:1257S-62S. [PubMed: 19403705]

15. Kneuss RM, Christiansen MP, Griffen SC, Keim NL, Havel PJ. Consumption of fructosesweetened beverages for 10 weeks in postprandial triacylglycerol and apolipoprotein B concentration in overweight and obese women. Br J Nutr. 2008; 100:947-52. [PubMed: 18384705]

16. Akerblom HK, Siltanen I, Kallio AK. Does dietary fructose affect the control of diabetes in children? Acta Med Scand Suppl. 1972; 542:187-193. [PubMed: 4579750]

17. The SEARCH Study Group. SEARCH for Diabetes in Youth: A multicenter study of the prevalence, incidence and classification of diabetes mellitus in youth. Control Clin Trials. 2004; 25:458-471. [PubMed: 15465616] 
18. Block, G.; Murphy, M.; Roullet, J.; Wakimoto, P.; Crawford, P.; Block, T. Pilot validation of a FFQ for children 8-10 years; Paper presented at: Fourth International Conference on Dietary Assessment Methods; September 17, 2000; Tucson, AZ.

19. Mayer-Davis RJ, Nichols M, Liese AD, Bell RA, Dabelea DM, Johansen JM, et al. the SEARCH for Diabetes in Youth Study Group. J Am Diet Assoc. 2006; 106:689-697. [PubMed: 16647326]

20. Hainline A Jr, Miller DT, Mather A. The Coronary Drug Project. Role and methods of the Central Laboratory. Controlled Clin Trials. 1983; 4:377-382. [PubMed: 6327187]

21. Kuczmarski RJ, Ogden CL, Guo SS, Grummer-Strawn LM, Flegal KM, Mei Z, et al. 2000 CDC Growth Charts for the United States: methods and development. Vital Health Stat. 2002; 246:1190.

22. Brenar ND, Kann L, Kinchen SA, Grunbaum JA, Whalen L, Eaton D. Methodology of the youth risk behavior surveillance system. MMWR Recomm Rep. 2004; 53:1-13.

23. Willett WC, Howe GR, Kushi LH. Adjustment for total energy intake in epidemiology studies. Am J Clin Nutr. 1997; 65:1220S-8S. [PubMed: 9094926]

24. United States Department of Agriculture. Nutrient Database for Standard Reference. Accessed at http://ndb.nal.usda.gov/ on November 15, 2012

25. Curry DL. Effects of mannose and fructose on the synthesis and secretion of insulin. Pancreas. 1989; 4:2-9. [PubMed: 2654926]

26. Frayn EA, Kingman SM. Dietary sugars and lipid metabolism in humans. Am J Clin Nutr. 1995; 62(Suppl):250S-61S. [PubMed: 7598082]

27. Storlien LH, Oakes ND, Pan DA, Kusunoki M, Jenkins AB. Syndromes of insulin resistance in the rat. Inducement by diet and amelioration with benfluorex Diabetes. 1993; 42:457-62.

28. Herman RH, Zakim D, Stifel FB. Effect of diet on lipid metabolism in experimental animals and man. Fed Proc. 1970; 29:1302-7. [PubMed: 4245709]

29. Okazaki M, Zhang H, Yoshida Y, Ichino K, Nakayama S, Oguchi K. Correlations between plasma fibrinogen and serum lipids in rats with hyperlipidemia induced by cholesterol free-high fructose or high cholesterol diet. J Nutr Sci Vitaminol. 1994; 40:479-89. [PubMed: 7891208]

30. Elliott SS, Keim NL, Stern JS, Teff K, Havel PJ. Fructose, weight gain, and the insulin resistance syndrome. Am J Clin Nutr. 2002; 76:911-22. [PubMed: 12399260]

31. Pollack NK, Bundy V, Kanto W, Davis CL, Bernard PJ, Zhu H, Gutin B, Dong Y. Greater fructose consumption is associated with cardiometabolic risk markers and visceral adiposity in adolescents. J Nutr. 2012; 142:251-257. [PubMed: 22190023]

32. Aeberli I, Zimmermann M, Molinari L. Fructose intake as a predictor of LDL particle size in overweight schoolchildren. Am J Clin Nutr. 2007; 86:1174-1178. [PubMed: 17921399]

33. Bortsov AV, Liese AD, Bell RA, Dabelea D, D'Agostino RB, Hamman RF, et al. Sugar-sweetened and diet beverage consumption is associated with cardiovascular risk facto profile in youth with type 1 diabetes. Acta Diabetologica. 2011; 48:275-82. [PubMed: 21249401]

34. Park YK, Yetley EA. Intakes and food sources of fructose in the United States. Am J Clin Nutr. 1993; 58:S737-47.

35. Livesey G, Taylor R. Fructose consumption and consequences for glycation, plasma triacylglycerol and body weight: meta-analyses and meta-regression models of intervention studies. Am J Clin Nutr. 2008; 88:1419-1437. [PubMed: 18996880]

36. Juhola J, Magnussen CG, Vikari JSA, Kahonen M, Hutri-Kahonen N, Jula A, Lehtimaki T, Akerblom HK, Pietikainen M, Laitinen T, Jokinen E, Taittonen L, Raitakari OT, Juonala M. Tracking of serum lipid levels, blood pressure, and body mass index from childhood to adulthood: The Cardiovascular Risk in Young Finns Study. J Pediatrics. 2011; 159:584-590.

37. Burrows TL, Martin RJ, Collins CE. A systematic review of the validity of dietary assessment methods in children when compared with the method of doubly labeled water. J Am Diet Assoc. 2010; 110:1501-1510. [PubMed: 20869489] 


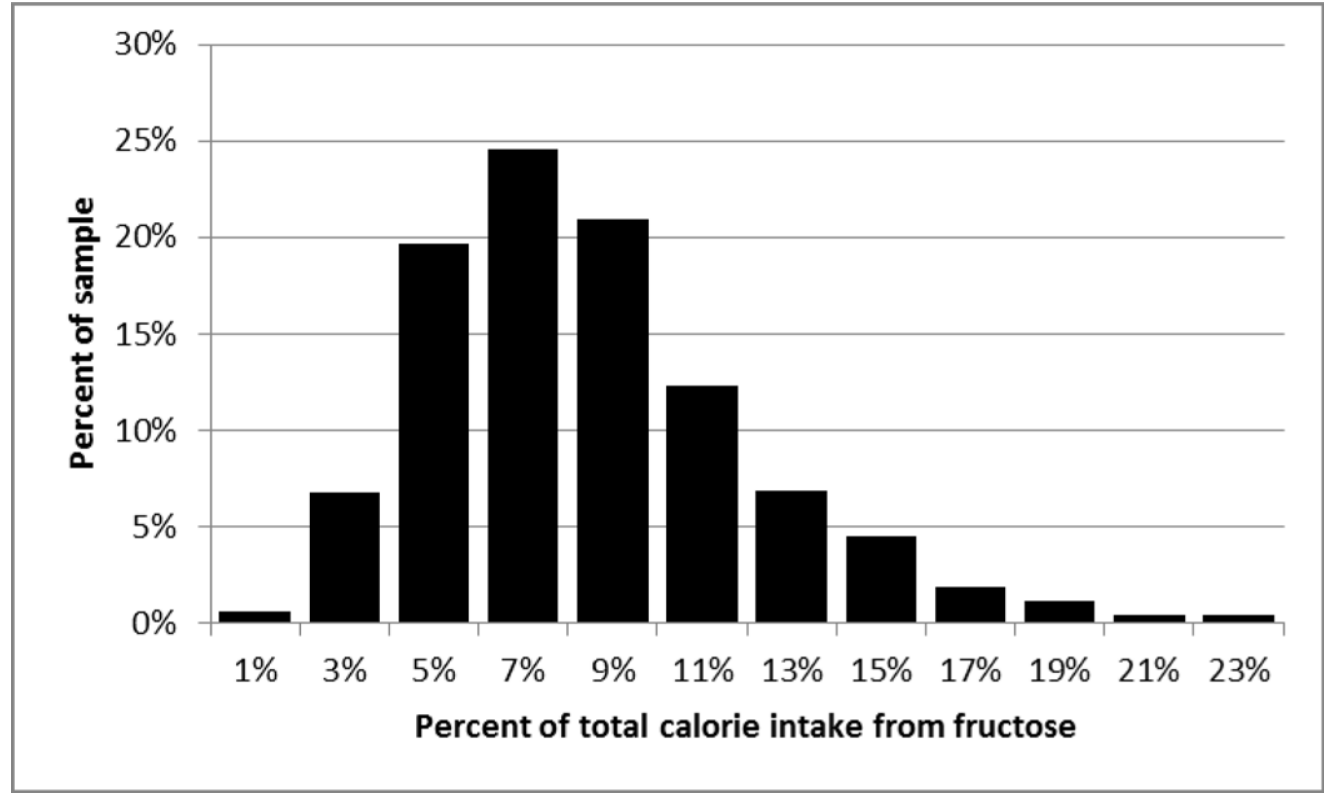

Figure 1.

Distribution of fructose intake (\% of total calories) in adolescents with T1DM: SEARCH for Diabetes in Youth. 


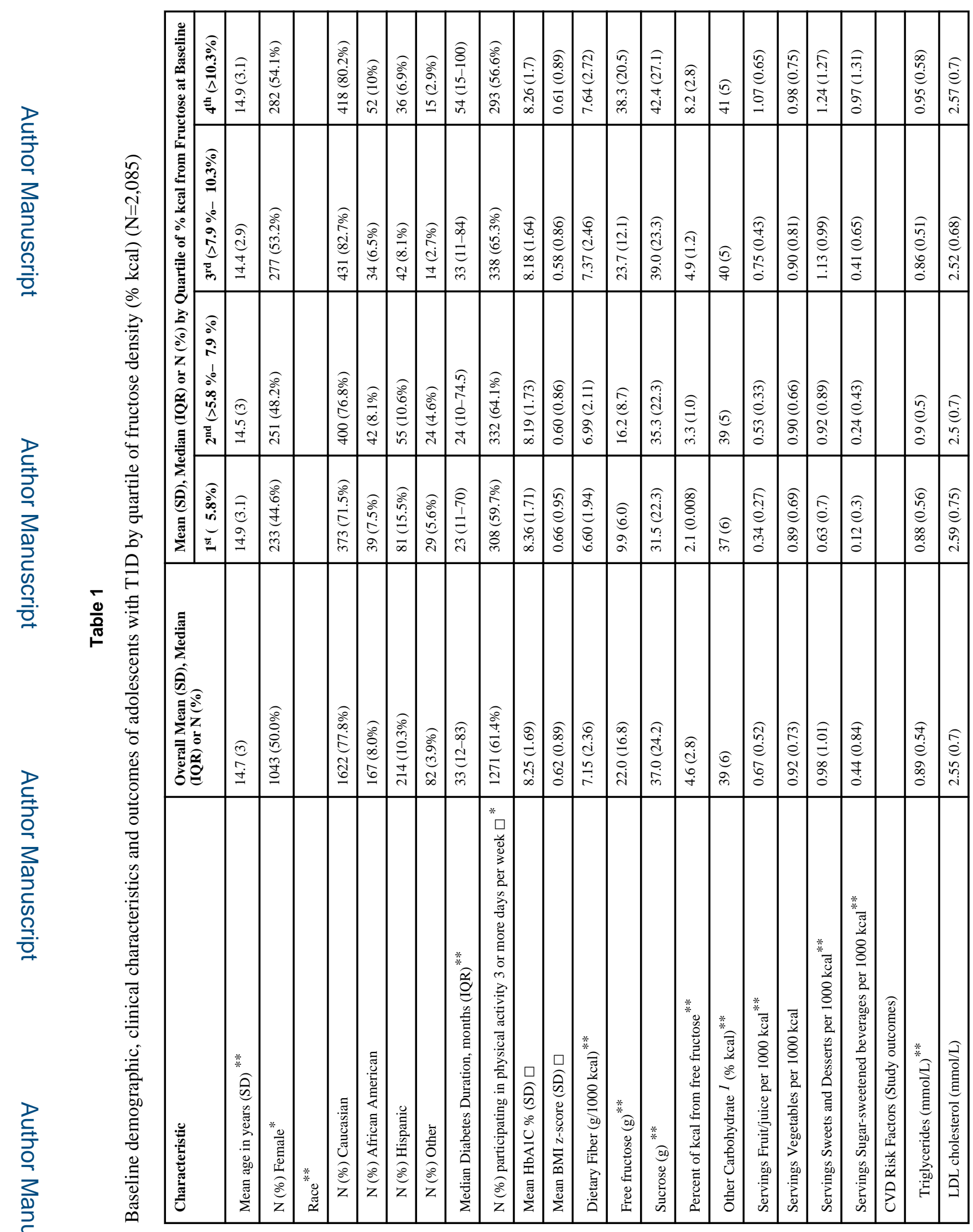

Diabetes Res Clin Pract. Author manuscript; available in PMC 2014 May 01. 


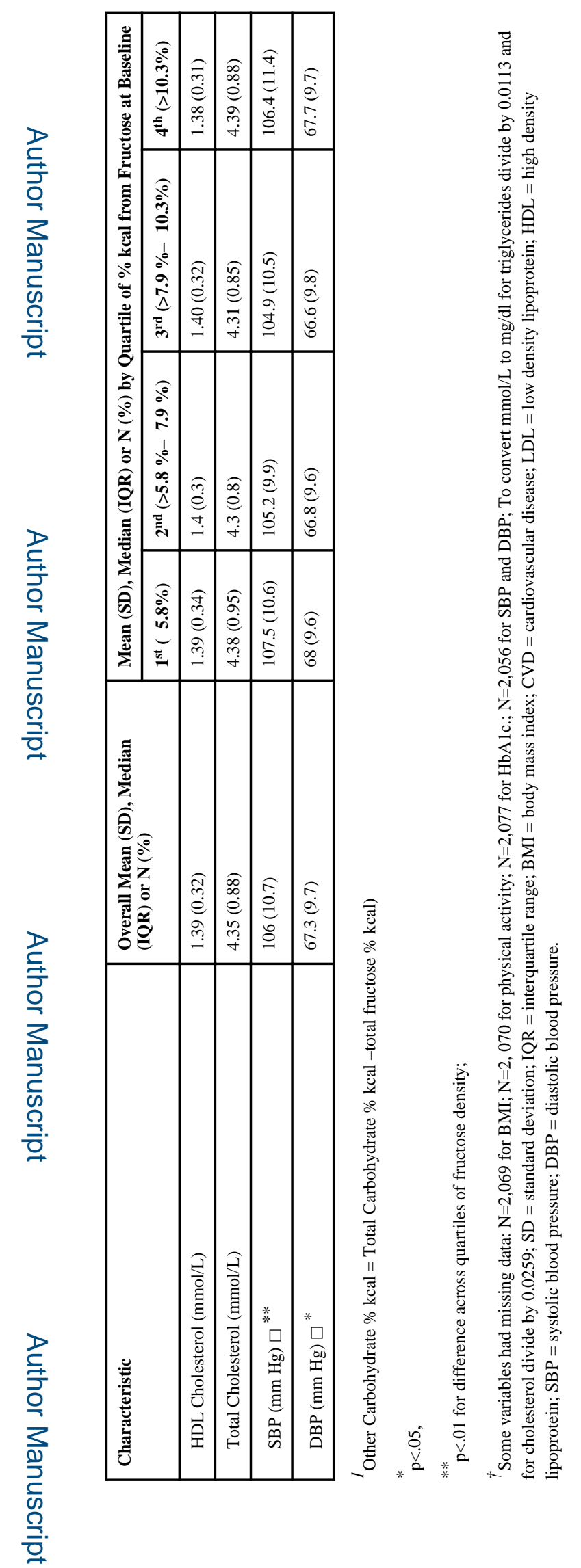

Diabetes Res Clin Pract. Author manuscript; available in PMC 2014 May 01. 


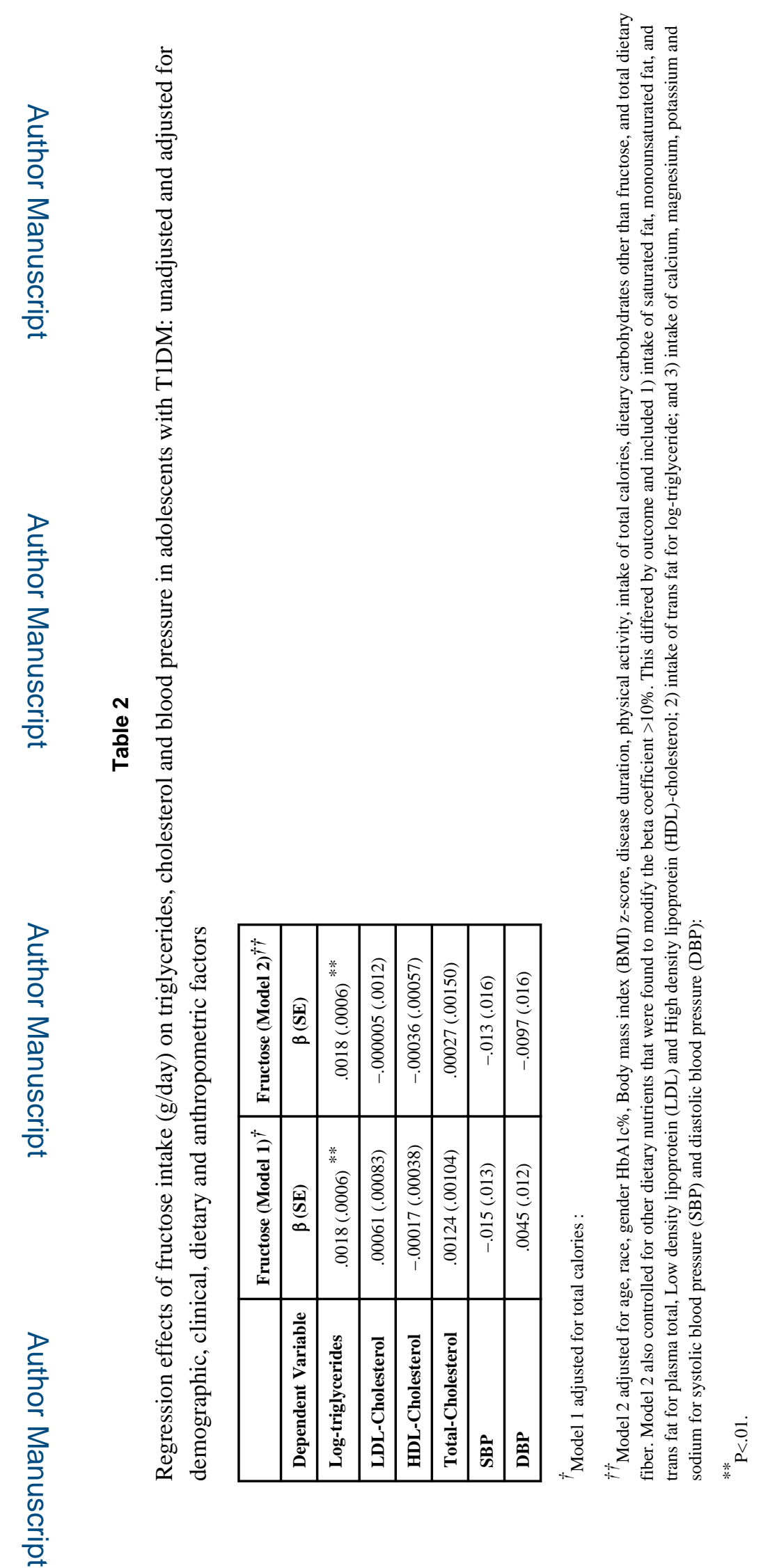

Diabetes Res Clin Pract. Author manuscript; available in PMC 2014 May 01. 